\title{
Effects of Cooking Methods on Free Amino Acid Contents in Vegetables
}

\author{
Hinako Ito ${ }^{1}$, Hiroe KIKUZAKI ${ }^{1}$ and Hiroshi $\mathrm{UENO}^{2, *}$ \\ ${ }^{1}$ Graduate School of Humanities and Sciences, Nara Women's University, \\ Kitauoyanishi-machi, Nara 630-8506, Japan \\ ${ }^{2}$ Laboratory of Applied Microbiology and Biochemistry, Faculty of Agriculture, \\ Ryukoku University, Seta-Ohe-cho, Shiga 520-2194, Japan
}

(Received September 27, 2018)

\begin{abstract}
Summary Vegetables are rich sources of nutrients such as fiber, minerals, vitamins, and antioxidants. Vegetables also contain various free-form amino acids, which improves their nutritional and palatable value. Cooking alters the content of free amino acids in vegetables, which affects their nutritional values. In this study, free amino acid levels were evaluated after cooking vegetables by different methods, boiling, roasting in an oven, and using a microwave. Results showed that many vegetables analyzed contain aspartate and glutamine abundantly. On the other hand, hydroxyproline, cysteine, ornithine and citrulline are the free amino acids existing at low or undetectable levels in all vegetables tested. The total free amino acid content in vegetables tended to decrease after boiling, and almost the same amount of free amino acids was obtained in the cooking liquid. Roasting of vegetables in an oven resulted in an increase in the content of specific amino acids, including $\gamma$-aminobutyric acid (GABA). Thus, it is important to choose the right cooking methods to prevent the loss of free amino acids. The results of the present study emphasize the changes in the contents of free amino acids during cooking with methods that are typically used on a daily basis. Our study on the dynamics of free amino acids caused by various cooking methods provides ample information for future nutritional studies.
\end{abstract}

Key Words HPLC, amino acid analysis, NBD-F, thermal cooking process, cooking liquid

Vegetables are rich sources of nutrients such as fiber, minerals, vitamins, and antioxidants. In addition, free amino acids are present in vegetables. As vegetables contain various nutrients, they serve as one of the most necessary components of a diet. Vegetables are usually cooked before eating, although some are eaten raw. The increase in digestibility often facilitates absorption of nutrients. Cooking vegetables by heating is used for the purpose of food safety, as heat tends to inhibit microbial growth. However, some nutrients such as polyphenols, glucosinolates, vitamins, and chlorophyll are reported to be lost during cooking (1-4).

Free amino acids in foods are sensitive to cooking methods. Raw rice undergoes significant alterations in the contents of glutamate (Glu) and aspartate (Asp) upon steaming (5). Tomato roasted in an oven showed an increase in the level of free amino acids such as $\gamma$-aminobutyric acid (GABA) and Glu. This observation was associated with specific enzyme reactions in response to the rise in temperature (6). Roasting tends to raise Glu contents of vegetables and imparts better umami and taste (6). Three different cooking methods, boiling, steaming, and stir-frying, were applied to raw bamboo shoot. Analysis of free amino acid contents of cooked bamboo shoot revealed that the composition of

\footnotetext{
*To whom correspondence should be addressed.

E-mail: ueno@agr.ryukoku.ac.jp
}

free amino acids was different among samples subjected to different cooking methods (7). In comparison with other methods, steam cooking resulted in an increase in the content of glycine (Gly) and Glu.

The understanding of the dynamics of free amino acid contents and different cooking methods for vegetables is important for proper utilization of cooked vegetables as a source of nutrients. There have been many studies targeting the dynamics of specific amino acids like Glu during cooking, which are expected to affect the health of humans (6); however, few studies have investigated these dynamics of the more than 20 types of free amino acids. In this study, changes in the levels of 25 different free amino acids induced by cooking were evaluated to clarify the most suitable cooking method for the palatability and nutrition of vegetables. This study's results will contribute to the free amino acid database, which is under construction $(8,9)$

\section{MATERIALS AND METHODS}

Cooking methods and vegetables. For cooking methods, we referred to the Standards Tables of Food Composition in Japan 2015 (http://www.mext.go.jp/a_menu/ syokuhinseibun/1365297.htm). According to this table, various ingredients such as seasonings and oils are added; however, in this study, no seasonings or oils were added to eliminate any factor that might influence the composition of free amino acids during cooking. 
Table 1. Cooking process of vegetables.

\begin{tabular}{|c|c|c|c|c|c|c|}
\hline & \multirow[b]{2}{*}{ Vegetables } & \multirow[b]{2}{*}{ Process } & \multirow[b]{2}{*}{$\begin{array}{l}\text { Cooking } \\
\text { time (min) }\end{array}$} & \multirow[b]{2}{*}{ Sample treatment } & \multicolumn{2}{|c|}{ Weight of vegetable (g) } \\
\hline & & & & & Before cooking & $\begin{array}{l}\text { After cooking } \\
\text { (weight change) }\end{array}$ \\
\hline \multirow[t]{3}{*}{1} & Western carrot & Raw & - & cut size $4.0 \mathrm{~cm} \times$ about $1.0 \mathrm{~cm}$ & - & \\
\hline & & Boiling & 9 & cut size $4.0 \mathrm{~cm} \times$ about $1.0 \mathrm{~cm}$ & 62.6 & $62.2(99.4 \%)$ \\
\hline & & Oven cooking & 15 & cut size $4.0 \mathrm{~cm} \times$ about $1.0 \mathrm{~cm}$ & 56.2 & $52.3(93.1 \%)$ \\
\hline \multirow[t]{2}{*}{2} & Kintoki carrot & Raw & - & cut size $4.0 \mathrm{~cm} \times$ about $1.0 \mathrm{~cm}$ & & \\
\hline & & Boiling & 12 & cut size $4.0 \mathrm{~cm} \times$ about $1.0 \mathrm{~cm}$ & 63.5 & $63.1(99.4 \%)$ \\
\hline \multirow[t]{2}{*}{3} & Sweet potato & Raw & - & cut size $1.0 \mathrm{~cm}$ slice & & \\
\hline & & Oven cooking & 105 & cut size $1.0 \mathrm{~cm}$ slice & 90.5 & $85.2(94.1 \%)$ \\
\hline \multirow[t]{2}{*}{4} & Snap pea & Raw & - & remove stem end and strings & & \\
\hline & & Boiling & 2 & remove stem end and strings & 83.6 & $83.5(99.9 \%)$ \\
\hline \multirow[t]{3}{*}{5} & Cabbage & Raw & - & divided by leaf & & \\
\hline & & Boiling & 2 & divided by leaf & 88.5 & $84.0(94.9 \%)$ \\
\hline & & Microwave & 2 & divided by leaf & 86.7 & $86.0(99.2 \%)$ \\
\hline \multirow[t]{2}{*}{6} & Spinach & Raw & - & roots removed & & \\
\hline & & Boiling & 2 & roots removed & 165.0 & $123.2(74.7 \%)$ \\
\hline \multirow[t]{2}{*}{7} & Komatsuna & Raw & - & roots removed & & \\
\hline & & Boiling & 3 & roots removed & 78.3 & $59.5(76.0 \%)$ \\
\hline
\end{tabular}

Cooking with a microwave is useful way of reducing vitamins loss and cooking in a short time. It has been reported that a biologically active compound in red cabbage was increased by microwave treatment (10). In this research, cabbage is examined with microwave treatment in addition to boiling. Vegetables used in this research were purchased from supermarkets in Shiga Prefecture or through mail order. The process and treatment of 7 vegetables are shown in Table 1. Details of the treatment of vegetables are as follows:

1) Western carrot: Western carrot was purchased from a supplier in Kochi Prefecture. Three samples, namely, boiled, oven cooked (roasted), and raw were tested. Fresh Western carrot was peeled, washed clean, and vertically cut into $4.0 \mathrm{~cm}$ sticks. The samples were weighed and stored at $-20^{\circ} \mathrm{C}$ as raw samples. For boiling, the sliced samples were placed in a pot filled with water and boiled for $9 \mathrm{~min}$. Boiled samples were scooped up and cooled to room temperature. For oven cooking, cut samples were placed in the oven at $200^{\circ} \mathrm{C}$ for $15 \mathrm{~min}$ after the preheating of oven to $200^{\circ} \mathrm{C}$.

2) Kintoki carrot: Kintoki carrot was purchased from a supplier in Kyoto Prefecture and divided into raw and boiled samples. Fresh Kintoki carrot was first peeled, washed clean, and vertically cut into $4.0 \mathrm{~cm}$ sticks. A part of the carrots was weighed and stored at $-20^{\circ} \mathrm{C}$ as a raw sample, while the rest was placed into a pot filled with water and boiled for $12 \mathrm{~min}$. These samples were scooped up and cooled to room temperature.

3) Sweet potato: Sweet potato was purchased from a supplier in Shiga Prefecture and was divided into raw and oven cooked samples. Fresh sweet potato was peeled, washed clean, and vertically cut into slices with about $1.0 \mathrm{~cm}$ thickness. The sliced samples were weighed and stored at $-20^{\circ} \mathrm{C}$ as raw samples. Another group of samples was placed in an oven preheated to $160^{\circ} \mathrm{C}$ and roasted for $90 \mathrm{~min}$. The oven temperature was then lowered to $150^{\circ} \mathrm{C}$ and the samples were heated for an additional $15 \mathrm{~min}$.

4) Snap pea: Snap peas were purchased from a supplier in Ehime Prefecture and divided into raw and boiled samples. The stem end and strings were removed from the fresh snap peas and discarded. The samples were weighed and stored at $-20^{\circ} \mathrm{C}$ as raw samples. Another group of samples was placed into a pot filled with hot water and then boiled for $2 \mathrm{~min}$.

5) Cabbage: Cabbage was purchased from a supplier in Ehime Prefecture and divided into raw, boiled, and microwaved samples. Fresh cabbage was firstly washed clean and then divided by leaf. The samples were weighed and stored at $-20^{\circ} \mathrm{C}$ as raw samples. For boiling, a raw sample was placed into a pot filled with hot water and then boiled for $2 \mathrm{~min}$. The sample weight after boiling was $95 \%$ of that before boiling. Microwave cooking was carried out by placing the sample in a heatresistant container, followed by heating at $700 \mathrm{~W}$ for 2 min.

6) Spinach: Spinach was purchased from a supplier in Fukuoka Prefecture and divided into raw and boiled samples. Fresh spinach was washed clean and the roots removed. The samples were weighed and stored at $-20^{\circ} \mathrm{C}$ as raw samples. For boiling, raw samples were placed in a pot filled with hot water and then boiled for 2 min.

7) Komatsuna: Komatsuna was purchased from a Fukuoka Prefecture supplier and divided into raw and boiled samples. Fresh komatsuna was washed clean and the roots removed. The samples were weighed and stored at $-20^{\circ} \mathrm{C}$ as raw samples. For boiling, raw samples were placed in a pot filled with hot water and then boiled for $3 \mathrm{~min}$.

The ingredients were detected in the boiled water 
Table 2. Free amino acid contents of raw and cooked Western carrots $^{1}(\mu \mathrm{mol} / 100 \mathrm{~g})$.

\begin{tabular}{|c|c|c|c|}
\hline & $\begin{array}{c}\text { Raw } \\
\text { Means } \pm \text { SE }\end{array}$ & $\begin{array}{c}\text { Boiled } \\
\text { Means } \pm \text { SE }\end{array}$ & $\begin{array}{r}\text { Roasted } \\
\text { Means } \pm \text { SE }\end{array}$ \\
\hline His & $9.572 \pm 0.844^{\mathrm{a}}$ & nd & $8.784 \pm 0.281^{\mathrm{a}}$ \\
\hline Arg & $10.95 \pm 1.002^{\mathrm{a}}$ & nd & $12.57 \pm 1.625^{\mathrm{a}}$ \\
\hline HYP & nd & nd & nd \\
\hline Asn & $196.3 \pm 9.586^{a}$ & $55.94 \pm 6.867^{b}$ & $214.6 \pm 13.53^{\mathrm{a}}$ \\
\hline Gln & $394.1 \pm 16.26^{\mathrm{a}}$ & $97.08 \pm 12.93^{b}$ & $344.7 \pm 34.29^{\mathrm{a}}$ \\
\hline Cit & nd & nd & nd \\
\hline Ser & $80.57 \pm 4.213^{a}$ & $23.15 \pm 0.960^{b}$ & $70.76 \pm 4.507^{\mathrm{a}}$ \\
\hline Asp & $101.9 \pm 5.385^{\mathrm{a}}$ & $53.10 \pm 4.080^{\mathrm{b}}$ & $89.14 \pm 3.984^{\mathrm{a}}$ \\
\hline Glu & $103.8 \pm 4.573^{\mathrm{a}}$ & $48.81 \pm 1.834^{\mathrm{b}}$ & $122.1 \pm 5.100^{\mathrm{a}}$ \\
\hline Thr & $13.51 \pm 0.758^{a}$ & $4.384 \pm 0.243^{\mathrm{b}}$ & $13.32 \pm 0.608^{a}$ \\
\hline Pro $+\beta$-Ala & $37.44 \pm 2.824^{\mathrm{a}}$ & $5.930 \pm 0.454^{\mathrm{b}}$ & $29.95 \pm 5.543^{\mathrm{a}}$ \\
\hline Gly & $6.529 \pm 0.574^{\mathrm{a}}$ & nd & $7.333 \pm 0.360^{\mathrm{a}}$ \\
\hline GABA & $60.63 \pm 3.834^{\mathrm{a}}$ & $9.963 \pm 0.534^{b}$ & $75.88 \pm 9.702^{\mathrm{a}}$ \\
\hline Ala & $192.2 \pm 14.12^{\mathrm{a}}$ & $74.29 \pm 5.054^{b}$ & $224.6 \pm 9.691^{\mathrm{a}}$ \\
\hline Val & $57.50 \pm 2.692^{\mathrm{a}}$ & $17.67 \pm 0.849^{b}$ & $46.13 \pm 4.165^{\mathrm{a}}$ \\
\hline Met & $10.24 \pm 0.813^{a}$ & nd & $6.027 \pm 0.212^{b}$ \\
\hline Leu & $22.50 \pm 3.290^{\mathrm{a}}$ & $14.07 \pm 4.117^{\mathrm{b}}$ & $24.76 \pm 6.177^{\mathrm{a}}$ \\
\hline Ile & $26.45 \pm 1.093^{\mathrm{a}}$ & $8.534 \pm 0.403^{b}$ & $21.72 \pm 1.884^{\mathrm{a}}$ \\
\hline Trp & nd & $2.835 \pm 0.182^{\mathrm{a}}$ & nd \\
\hline Orn & nd & nd & nd \\
\hline Phe & $31.64 \pm 2.329^{a}$ & $9.402 \pm 0.795^{b}$ & $21.38 \pm 0.903^{\mathrm{a}}$ \\
\hline Lys & nd & nd & nd \\
\hline Cys & nd & nd & nd \\
\hline Tyr & $12.24 \pm 0.486^{\mathrm{a}}$ & $5.927 \pm 0.364^{b}$ & $9.290 \pm 0.910^{\mathrm{a}}$ \\
\hline TFAA & $1,368.1 \pm 54.29^{\mathrm{a}}$ & $431.1 \pm 28.88^{b}$ & $1,343.0 \pm 72.53^{\mathrm{a}}$ \\
\hline
\end{tabular}

${ }^{1}$ In all of the cooking methods, the value is shown in terms of the amount of per $100 \mathrm{~g}$ raw state.

Data area presented as mean $\pm \operatorname{SE}(n=3)$.

TFAA: total free amino acids, nd: not detected.

In Table 2 and the following figures, the values are converted in the same way.

Mean values with different letters, a and $b$, in the same row are significantly different $(p<0.05)(7)$.

Abbreviations of amino acid analyzed (Three letter codes): 1. histidine (His), 2. arginine (Arg), 3. hydroxyproline (HYP), 4. asparagine (Asn), 5. glutamine (Gln), 6. citrulline (Cit), 7. serine (Ser), 8. aspartate (Asp), 9. glutamate (Glu), 10. threonine (Thr), 11. proline $+\beta$-alanine (Pro $+\beta$-Ala), 12. glycine (Gly), 13. $\gamma$-aminobutyric acid (GABA), 14. alanine (Ala), 15. valine (Val), 16. methionine (Met), 17. leucine (Leu), 18. isoleucine (Ile), 19. tryptophan (Trp), 20. ornithine (Orn), 21. phenylalanine (Phe), 22. lysine (Lys), 23. cysteine (Cys), 24. tyrosine (Tyr), total free amino acids (TFAA).

(cooking liquid) upon boiling of the vegetables. Hence, cooking liquid samples from snap pea, cabbage, spinach, and komatsuna were saved and used for amino acid analyses.

Extraction of free amino acids from vegetables. About $10 \mathrm{~g}$ of frozen samples were treated with $100 \mathrm{~mm}$ HEPES-Na buffer, pH 7.0, and homogenized on ice with a Tissue-Tearor (Biospec). The homogenate was centrifuged at $10,000 \times g$ for $15 \mathrm{~min}$ and the supernatant was collected. Proteins in the supernatant were removed by adding $60 \%$ perchloric acid and the precipitate was separated by centrifugation. The protein content of samples was estimated by Bradford protein assay (Bio-Rad). Samples were subjected to ultrafiltration using CENTRI$\mathrm{CON}^{\circledR} 10$ (Millipore) to remove any remaining proteins. After ultrafiltration, protein content was measured with the Bradford protein assay.

Amino acid standards. GABA, L-hydroxyproline (HYP), L-Gln, L-Asn, $\beta$-alanine ( $\beta$-Ala), L-cysteine
(L-Cys), L-ornithine (L-Orn), L-citrulline (L-Cit), and L-tryptophan (L-Trp) were purchased from Wako Pure Chemical Industries, Ltd. These amino acids were added to type $\mathrm{H}$ amino acid mixture standard solution (Wako Pure Chemical Industries, Ltd.) to produce a working standard solution of 25 amino acids, wherein the concentration of each amino acid was $100 \mu \mathrm{mol} / \mathrm{L}$ in $0.1 \mathrm{~N}$ hydrochloric acid $(\mathrm{HCl})$.

Amino acid analysis. Amino acid samples were treated with 4-fluoro-7-nitrobenzofurazan (NBD-F) and the derivatized NBD-amino acids were separated on a Zorbax SB-C18 column $(3.0 \times 50 \mathrm{~mm}, 2.7 \mu \mathrm{m}$; Agilent Technologies) attached to a Hitachi Ultra High-Speed Liquid Chromatography system equipped with UV-Vis detector monitoring at $470 \mathrm{~nm}$, or a Shimadzu Nexera XR Liquid Chromatography system equipped with a UV-VIS detector. Derivatization of amino acids was performed according to the Hitachi Ultra High-Speed Liquid Chromatography manual. NBD-amino acids were 
Table 3. Free amino acid contents of raw and cooked Kintoki carrots $(\mu \mathrm{mol} / 100 \mathrm{~g})$.

\begin{tabular}{|c|c|c|}
\hline & $\begin{array}{c}\text { Raw } \\
\text { Means } \pm \text { SE }\end{array}$ & $\begin{array}{c}\text { Boiled } \\
\text { Means } \pm \text { SE }\end{array}$ \\
\hline His & $5.380 \pm 0.300$ & $4.172 \pm 0.242$ \\
\hline Arg & $4.012 \pm 0.258$ & $3.843 \pm 0.286$ \\
\hline HYP & nd & nd \\
\hline Asn & $219.3 \pm 36.48$ & $142.3 \pm 8.564^{*}$ \\
\hline Gln & $967.5 \pm 121.3$ & $596.7 \pm 34.86^{*}$ \\
\hline Cit & nd & nd \\
\hline Ser & $53.28 \pm 4.883$ & $34.98 \pm 1.672 *$ \\
\hline Asp & $40.03 \pm 2.353$ & $34.97 \pm 2.583$ \\
\hline Glu & $48.74 \pm 4.599$ & $40.91 \pm 2.932$ \\
\hline Thr & $34.03 \pm 3.760$ & $8.455 \pm 0.524$ \\
\hline Pro $+\beta$-Ala & $11.70 \pm 0.611$ & $6.379 \pm 0.340^{*}$ \\
\hline Gly & $10.64 \pm 1.380$ & $9.493 \pm 0.582$ \\
\hline GABA & $27.63 \pm 2.717$ & $14.96 \pm 1.466^{*}$ \\
\hline Ala & $104.9 \pm 14.94$ & $87.79 \pm 3.807$ \\
\hline Val & $24.90 \pm 2.866$ & $18.42 \pm 1.310$ \\
\hline Met & $2.522 \pm 0.000$ & nd \\
\hline Leu & $6.684 \pm 1.419$ & $9.692 \pm 1.646$ \\
\hline Ile & $5.520 \pm 0.605$ & $4.597 \pm 0.347$ \\
\hline Trp & nd & nd \\
\hline Orn & nd & nd \\
\hline Phe & $5.592 \pm 0.258$ & $3.983 \pm 0.301$ \\
\hline Lys & nd & nd \\
\hline Cys & nd & nd \\
\hline Tyr & $3.186 \pm 0.195$ & $5.884 \pm 0.329^{*}$ \\
\hline TFAA & $1,575.5 \pm 232.2$ & $1,027.5 \pm 57.71$ \\
\hline
\end{tabular}

$* p<0.05$.

eluted using a gradient of increasing elution solution B as follows: equilibration with $15 \%, 15-25 \%$ for $2.7 \mathrm{~min}$, $25-35 \%$ for $3.5 \mathrm{~min}, 35-70 \%$ for $1.0 \mathrm{~min}, 70-85 \%$ for $0.1 \mathrm{~min}$, and $85 \%$ for $1.5 \mathrm{~min}$. Elution solutions A and B were purchased from Hitachi High Technologies. The flow rate was maintained at $0.550 \mathrm{~mL} / \mathrm{min}$ throughout the analysis.

Each food sample was analyzed thrice per extraction and the average values were used for analysis. The analytical system was stable and highly reproducible as previously reported, except for proline and $\beta$-alanine, which showed coelution (9).

During the comparison of free amino acid dynamics between raw and cooked samples, significantly large changes in weight were observed; hence, cooked samples were expressed in terms of the amount of free amino acids per $100 \mathrm{~g}$ of raw material $(\mu \mathrm{mol} / 100 \mathrm{~g})$. The cooking liquid was converted from the amount of cooking liquid and the amount of boiled sample to the amount of free amino acids per $100 \mathrm{~g}$ of raw weight.

Amount of free amino acids eluted from vegetables during boiling ( $\mu \mathrm{mol} / 100 \mathrm{~g}$ )

$=$ Free amino acid concentration of cooking liquid $(\mu \mathrm{mol} / \mathrm{L}) \times$ boiling water volume $(\mathrm{L}) /$ sample weight $(\mathrm{g}) \times 100 \mathrm{~g}$

For comparison of the composition of free amino acids in vegetables between raw and cooked samples,
Table 4. Free amino acid contents of raw and cooked sweet potatoes $(\mu \mathrm{mol} / 100 \mathrm{~g})$.

\begin{tabular}{lcc}
\hline & $\begin{array}{c}\text { Raw } \\
\text { Means } \pm \text { SE }\end{array}$ & $\begin{array}{c}\text { Roasted } \\
\text { Means } \pm \text { SE }\end{array}$ \\
\hline His & $15.94 \pm 0.595$ & $14.57 \pm 0.991$ \\
Arg & $9.778 \pm 0.236$ & $9.089 \pm 0.578$ \\
HYP & nd & nd \\
Asn & $220.6 \pm 13.85$ & $145.1 \pm 13.41^{*}$ \\
Gln & $14.52 \pm 0.481$ & nd \\
Cit & nd & nd \\
Ser & $174.6 \pm 7.703$ & $128.7 \pm 9.905^{*}$ \\
Asp & $204.3 \pm 10.07$ & $66.67 \pm 5.581^{*}$ \\
Glu & $144.4 \pm 5.707$ & $96.24 \pm 8.276^{*}$ \\
Thr & $36.54 \pm 0.707$ & $28.16 \pm 1.811^{*}$ \\
Pro $+\beta$-Ala & $22.30 \pm 0.905$ & $23.63 \pm 1.284$ \\
Gly & $18.97 \pm 0.776$ & $21.88 \pm 1.112$ \\
GABA & $5.299 \pm 0.561$ & $22.81 \pm 2.300^{*}$ \\
Ala & $34.71 \pm 1.900$ & $107.7 \pm 9.385^{*}$ \\
Val & $51.48 \pm 3.239$ & $46.98 \pm 3.326$ \\
Met & $12.04 \pm 0.353$ & $10.74 \pm 0.724$ \\
Leu & $29.97 \pm 2.072$ & $33.23 \pm 2.904$ \\
Ile & $30.68 \pm 2.302$ & $25.40 \pm 2.366$ \\
Trp & $9.646 \pm 0.742$ & $10.14 \pm 0.891$ \\
Orn & nd & nd \\
Phe & $114.4 \pm 6.220$ & $95.11 \pm 7.058$ \\
Lys & $4.496 \pm 0.313$ & $2.471 \pm 0.651$ \\
Cys & nd & nd \\
Tyr & $23.46 \pm 1.533$ & $29.55 \pm 1.821$ \\
TFAA & $1,178.1 \pm 55.26$ & $918.2 \pm 69.62$ \\
\hline & & \\
\hline & &
\end{tabular}

$* p<0.05$

data were analyzed by $t$-tests. A value of $p<0.05$ was considered significant. The significant difference in amino acids contents prepared at three cooking methods was resolved with 1-way ANOVA followed by Bonferroni correction by MS-Excel 2017.

Abbreviations of the amino acids analyzed are summarized in Table 2.

\section{RESULTS AND DISCUSSION}

Many raw vegetables analyzed contain Asn and Gln abundantly. On the other hand, HYP, Cys, Orn and Cit are the free amino acids existing at low or undetectable levels in all the vegetables tested.

Western carrot

Western carrot contained high levels of Gln, Asn, and Ala. Other amino acids were also detected, but the levels of His, Arg, Thr, Gly, Met, Trp, Lys, Cys, Orn, and Tyr were minor. The effect of oven cooking was not significant except for the decline of Met, but the contents of Asn, Glu, Ala, and GABA slightly increased in oven cooked samples as compared with raw samples (Table 2). However, boiled samples showed decreased levels of all analyzed amino acids as compared with raw samples, and a significant decrease in the total amount of free amino acids - actually one third of that of untreatedwas observed $(p<0.05)$. Because of this, boiling would reduce nutritional values as well as taste. 
Table 5. Free amino acid contents of raw and cooked snap peas $(\mu \mathrm{mol} / 100 \mathrm{~g})$.

\begin{tabular}{|c|c|c|c|}
\hline & $\begin{array}{c}\text { Raw } \\
\text { Means } \pm \text { SE }\end{array}$ & $\begin{array}{c}\text { Boiled } \\
\text { Means } \pm \text { SE }\end{array}$ & $\begin{array}{l}\text { Cooking liquid } \\
\text { Means } \pm \text { SE }\end{array}$ \\
\hline His & $51.68 \pm 3.501$ & $44.72 \pm 3.685$ & $5.134 \pm 0.384$ \\
\hline Arg & $44.57 \pm 4.457$ & $40.80 \pm 2.961$ & $2.122 \pm 0.113$ \\
\hline HYP & nd & nd & nd \\
\hline Asn & $1,065 \pm 86.80$ & $1,074 \pm 116.3$ & $95.83 \pm 0.143$ \\
\hline Gln & $916.0 \pm 115.1$ & $688.8 \pm 97.27$ & $106.9 \pm 2.020$ \\
\hline Cit & nd & nd & nd \\
\hline Ser & $528.8 \pm 40.41$ & $414.2 \pm 28.82$ & $87.37 \pm 1.498$ \\
\hline Asp & $74.51 \pm 7.549$ & $60.14 \pm 3.941$ & nd \\
\hline Glu & $53.71 \pm 4.742$ & $52.83 \pm 4.545$ & $12.70 \pm 0.303$ \\
\hline Thr & $161.5 \pm 16.86$ & $136.8 \pm 7.112$ & $28.10 \pm 0.267$ \\
\hline Pro $+\beta$-Ala & $82.02 \pm 11.14$ & $81.44 \pm 12.21$ & $10.05 \pm 0.131$ \\
\hline Gly & $17.45 \pm 1.500$ & $12.90 \pm 1.145$ & $5.185 \pm 0.407$ \\
\hline GABA & $24.51 \pm 2.351$ & $8.274 \pm 0.957^{*}$ & nd \\
\hline Ala & $338.1 \pm 33.41$ & $245.1 \pm 17.66$ & $59.94 \pm 1.107$ \\
\hline Val & $96.06 \pm 9.172$ & $80.12 \pm 4.396$ & $13.18 \pm 0.506$ \\
\hline Met & $6.890 \pm 1.090$ & $4.018 \pm 0.675$ & nd \\
\hline Leu & $20.65 \pm 1.957$ & $15.78 \pm 2.043$ & nd \\
\hline Ile & $35.67 \pm 2.630$ & $31.03 \pm 3.695$ & $8.545 \pm 0.115$ \\
\hline Trp & $2.224 \pm 0.096$ & $2.144 \pm 0.319$ & nd \\
\hline Orn & nd & nd & nd \\
\hline Phe & $20.60 \pm 1.945$ & $15.99 \pm 1.252$ & nd \\
\hline Lys & $13.64 \pm 4.056$ & $6.620 \pm 0.745$ & $3.318 \pm 0.173$ \\
\hline Cys & nd & nd & nd \\
\hline Tyr & $6.180 \pm 0.449$ & $10.98 \pm 0.648$ & $7.069 \pm 0.443$ \\
\hline TFAA & $3,559.8 \pm 186.7$ & $3,026.7 \pm 212.8$ & $445.4 \pm 6.287$ \\
\hline
\end{tabular}

${ }^{*} p<0.05$ (Between raw state and boiled state).

Table 6. Free amino acid contents of raw and cooked cabbage $(\mu \mathrm{mol} / 100 \mathrm{~g})$.

\begin{tabular}{|c|c|c|c|c|}
\hline & $\begin{array}{c}\text { Raw } \\
\text { Means } \pm \text { SE }\end{array}$ & $\begin{array}{c}\text { Boiled } \\
\text { Means } \pm \text { SE }\end{array}$ & $\begin{array}{l}\text { Cooking liquid } \\
\text { Means } \pm \text { SE }\end{array}$ & $\begin{array}{l}\text { Microwaved } \\
\text { Means } \pm S E\end{array}$ \\
\hline His & $52.51 \pm 5.714^{\mathrm{a}}$ & $15.02 \pm 1.054^{\mathrm{b}}$ & $12.52 \pm 0.223$ & $36.60 \pm 4.236^{\mathrm{a}}$ \\
\hline Arg & $55.00 \pm 5.988^{\mathrm{a}}$ & $18.09 \pm 1.818^{\mathrm{b}}$ & $15.32 \pm 1.193$ & $38.41 \pm 6.524^{\mathrm{a}}$ \\
\hline HYP & nd & nd & nd & nd \\
\hline Asn & $151.3 \pm 9.408^{\mathrm{a}}$ & $41.58 \pm 1.868^{\mathrm{b}}$ & $35.73 \pm 3.905$ & $97.75 \pm 11.49^{\mathrm{a}}$ \\
\hline Gln & $1,112 \pm 92.10^{\mathrm{a}}$ & $309.9 \pm 22.30^{\mathrm{b}}$ & $346.1 \pm 26.50$ & $751.0 \pm 100.8^{\mathrm{a}}$ \\
\hline Cit & nd & nd & nd & nd \\
\hline Ser & $159.4 \pm 6.489^{a}$ & $77.46 \pm 5.113^{b}$ & $81.90 \pm 6.279$ & $203.2 \pm 29.43^{\mathrm{a}}$ \\
\hline Asp & $39.08 \pm 2.857^{\mathrm{a}}$ & $12.28 \pm 0.875^{\mathrm{b}}$ & $18.43 \pm 1.779$ & $28.03 \pm 3.514^{\mathrm{a}}$ \\
\hline Glu & $74.82 \pm 4.368^{\mathrm{a}}$ & $27.75 \pm 0.845^{\mathrm{b}}$ & $37.85 \pm 2.074$ & $63.34 \pm 6.522^{\mathrm{a}}$ \\
\hline Thr & $45.17 \pm 1.039^{\mathrm{a}}$ & $18.52 \pm 0.846^{\mathrm{b}}$ & $19.61 \pm 5.138$ & $45.53 \pm 4.823^{\mathrm{a}}$ \\
\hline Pro $+\beta$-Ala & $315.5 \pm 29.45^{\mathrm{a}}$ & $95.02 \pm 0.934^{\mathrm{b}}$ & $79.83 \pm 4.479$ & $241.8 \pm 24.34^{\mathrm{a}}$ \\
\hline Gly & $29.40 \pm 0.810^{\mathrm{a}}$ & $17.36 \pm 2.434^{\mathrm{b}}$ & $18.77 \pm 0.961$ & $57.64 \pm 13.66^{\mathrm{a}}$ \\
\hline GABA & $25.01 \pm 3.136^{\mathrm{a}}$ & $4.383 \pm 0.530^{\mathrm{b}}$ & $5.136 \pm 0.847$ & $10.02 \pm 1.018^{b}$ \\
\hline Ala & $227.0 \pm 13.00^{\mathrm{a}}$ & $77.38 \pm 6.674^{\mathrm{b}}$ & $102.36 \pm 5.482$ & $218.9 \pm 37.82^{\mathrm{a}}$ \\
\hline Val & $54.38 \pm 2.158^{\mathrm{a}}$ & $20.23 \pm 0.832^{\mathrm{b}}$ & $27.53 \pm 1.527$ & $52.91 \pm 5.128^{\mathrm{a}}$ \\
\hline Met & $2.219 \pm 0.165^{\mathrm{a}}$ & nd & nd & nd \\
\hline Leu & $15.19 \pm 0.685^{\mathrm{a}}$ & $6.280 \pm 0.202^{\mathrm{b}}$ & $6.993 \pm 0.270$ & $14.97 \pm 1.644^{\mathrm{a}}$ \\
\hline Ile & $30.09 \pm 1.471^{\mathrm{a}}$ & $11.37 \pm 0.376^{b}$ & $23.89 \pm 0.725$ & $23.63 \pm 2.307^{\mathrm{a}}$ \\
\hline Trp & $4.358 \pm 0.196^{\mathrm{a}}$ & $2.328 \pm 0.069^{b}$ & $2.780 \pm 0.897$ & $6.437 \pm 0.972^{\mathrm{a}}$ \\
\hline Orn & nd & nd & nd & nd \\
\hline Phe & $13.15 \pm 0.347^{\mathrm{a}}$ & $4.945 \pm 0.155^{\mathrm{b}}$ & $6.446 \pm 0.815$ & $14.20 \pm 1.335^{\mathrm{a}}$ \\
\hline Lys & $7.674 \pm 1.030^{\mathrm{a}}$ & $3.655 \pm 0.120^{\mathrm{b}}$ & $4.852 \pm 0.394$ & $6.269 \pm 0.778^{\mathrm{a}}$ \\
\hline Cys & $2.891 \pm 0.100^{\mathrm{a}}$ & $2.300 \pm 0.082^{\mathrm{b}}$ & nd & $4.865 \pm 0.577^{\mathrm{a}}$ \\
\hline Tyr & $9.414 \pm 0.342^{\mathrm{a}}$ & $9.018 \pm 0.157^{\mathrm{a}}$ & $15.28 \pm 0.659$ & $16.66 \pm 1.038^{b}$ \\
\hline TFAA & $2,425.6 \pm 160.2^{\mathrm{a}}$ & $774.9 \pm 40.10^{\mathrm{b}}$ & $861.3 \pm 54.65$ & $1,932.2 \pm 189.7^{a}$ \\
\hline
\end{tabular}

Mean values with different letters, a and b, in the same row are significantly different $(p<0.05)(7)$. 
Table 7. Free amino acid contents of raw and cooked spinach $(\mu \mathrm{mol} / 100 \mathrm{~g})$.

\begin{tabular}{|c|c|c|c|}
\hline & $\begin{array}{c}\text { Raw } \\
\text { Means } \pm \text { SE }\end{array}$ & $\begin{array}{c}\text { Boiled } \\
\text { Means } \pm \text { SE }\end{array}$ & $\begin{array}{l}\text { Cooking liquid } \\
\text { Means } \pm \text { SE }\end{array}$ \\
\hline His & $19.56 \pm 1.695$ & $15.80 \pm 1.364$ & $10.20 \pm 0.779$ \\
\hline Arg & $9.061 \pm 1.227$ & $6.264 \pm 1.147^{*}$ & $5.629 \pm 0.416$ \\
\hline HYP & nd & nd & nd \\
\hline Asn & $62.63 \pm 5.269$ & $33.68 \pm 3.838^{*}$ & $18.42 \pm 0.527$ \\
\hline Gln & $347.3 \pm 30.09$ & $215.5 \pm 23.67^{*}$ & $138.0 \pm 7.493$ \\
\hline Cit & nd & nd & nd \\
\hline Ser & $64.61 \pm 8.841$ & $39.73 \pm 2.908$ & $20.70 \pm 2.456$ \\
\hline Asp & $40.72 \pm 7.485$ & $21.33 \pm 2.208^{*}$ & $12.76 \pm 0.480$ \\
\hline Glu & $67.29 \pm 10.79$ & $60.03 \pm 4.666$ & $36.67 \pm 0.234$ \\
\hline Thr & $26.28 \pm 2.660$ & $13.60 \pm 2.314^{*}$ & $7.042 \pm 0.074$ \\
\hline Pro $+\beta$-Ala & $58.85 \pm 13.48$ & $32.26 \pm 3.709$ & $18.44 \pm 1.062$ \\
\hline Gly & nd & nd & nd \\
\hline GABA & $16.60 \pm 3.368$ & nd & nd \\
\hline Ala & $21.29 \pm 3.417$ & $15.30 \pm 2.378$ & $12.57 \pm 0.122$ \\
\hline Val & $30.45 \pm 2.224$ & $22.16 \pm 2.719$ & $18.56 \pm 0.236$ \\
\hline Met & $2.831 \pm 0.272$ & nd & nd \\
\hline Leu & $29.68 \pm 3.786$ & $17.53 \pm 1.961$ & $19.11 \pm 0.179$ \\
\hline Ile & $17.16 \pm 1.625$ & $10.59 \pm 1.199$ & $16.17 \pm 0.672$ \\
\hline Trp & $15.48 \pm 1.221$ & $8.867 \pm 0.743^{*}$ & $6.125 \pm 0.385$ \\
\hline Orn & nd & nd & nd \\
\hline Phe & $20.49 \pm 1.590$ & $10.73 \pm 1.092^{*}$ & $10.74 \pm 0.785$ \\
\hline Lys & $7.451 \pm 1.500$ & $4.479 \pm 0.734$ & $4.237 \pm 0.149$ \\
\hline Cys & nd & $4.393 \pm 0.536$ & nd \\
\hline Tyr & $35.58 \pm 3.521$ & $28.12 \pm 1.910$ & $19.15 \pm 0.038$ \\
\hline TFAA & $893.3 \pm 86.11$ & $560.4 \pm 39.33^{*}$ & $374.5 \pm 16.09$ \\
\hline
\end{tabular}

${ }^{*} p<0.05$ (Between raw state and boiled state).

\section{Kintoki carrot}

Kintoki carrot contained abundant amounts of Gln (Table 3). The boiling treatment tended to reduce free amino acids; the total amount of free amino acids in boiled samples decreased to $65.2 \%$ as compared with raw samples. Individual amino acids, including Asn, Gln, and GABA, decreased to less than $60 \%(p<0.05)$ (Table 3). On the other hand, the contents of Tyr increased significantly after boiling $(p<0.05)$. The levels of Leu also tended to increase. The amount of TFAA tended to decrease to about $65 \%$.

Sweet potato

Sweet potatoes contained almost all types of free amino acids, with low levels of Lys and Cys (Table 4). After roasting, the total amount of free amino acids fell to about $80 \%$ of that reported in raw samples (Table 4). The levels of Asn, Asp, and Glu decreased to about $30 \%$ to $70 \%$, whereas Ala and GABA levels increased by about three times $(p<0.05)$. Gln level decreased after roasting. Eating sweet potatoes roasted in the oven would provide an ample amount of GABA.

Snap pea

Snap peas were rich in a few free amino acids in their raw state, including Asn, Gln, Ser, and Ala. The total amount of free amino acids after boiling was about 85\% of that reported in raw samples (Table 5). Effects of boiling were significant on the GABA level, which decreased $(p<0.05)$ after boiling; neither of the amino acids was detected in the cooking liquid. However, the levels of Asn, Pro (+ $\beta$-Ala), and Trp showed small changes. Eating boiled snap pea without cooking liquid would provide sufficient free amino acids except GABA.

\section{Cabbage}

Cabbage contained large amount of Gln, followed by Pro $(+\beta$-Ala), Ala, and Ser. After boiling, the total amount of free amino acids decreased to about $30 \%$ of that reported in raw samples. Boiling resulted in a significant reduction in the levels of almost all amino acids (Table 6). Examination of the cooking liquid revealed the loss of nearly all free amino acids after boiling. On the other hands, microwave cooking of cabbage retained about $80 \%$ of total free amino acids; Tyr in particular was increased after microwave cooking $(p<0.05)$; however, a significant loss in GABA was evident $(p<0.05)$. The loss of GABA in microwave cooking may be due to the unwanted break down of GABA probably caused by enzymatic reaction(s). The dietary intakes of not only boiled cabbage but also of cooking liquid like soups are recommended for the efficient utilization of free amino acids to improve palatability and their nutritional affect in the human body.

Spinach

Gln was the major free amino acid in spinach. Spinach contained almost every kind of amino acids. Effects of boiling on free amino acids in spinach were as follows: About $60 \%$ of the free amino acids were retained 
Table 8. Free amino acids contents of raw and cooked komatsuna ( $\mu \mathrm{mol} / 100 \mathrm{~g})$.

\begin{tabular}{|c|c|c|c|}
\hline & $\begin{array}{c}\text { Raw } \\
\text { Means } \pm \text { SE }\end{array}$ & $\begin{array}{c}\text { Boiled } \\
\text { Means } \pm \text { SE }\end{array}$ & $\begin{array}{l}\text { Cooking liquid } \\
\text { Means } \pm \text { SE }\end{array}$ \\
\hline His & $19.68 \pm 1.210$ & $9.199 \pm 1.098^{*}$ & $13.81 \pm 0.594$ \\
\hline Arg & $55.62 \pm 8.615$ & $19.51 \pm 2.958^{*}$ & $34.09 \pm 0.077$ \\
\hline HYP & nd & nd & nd \\
\hline Asn & $103.0 \pm 9.873$ & $35.81 \pm 7.286^{*}$ & $39.19 \pm 1.986$ \\
\hline Gln & $629.7 \pm 197.1$ & $308.8 \pm 87.16$ & $220.9 \pm 0.926$ \\
\hline Cit & nd & nd & nd \\
\hline Ser & $82.74 \pm 12.80$ & $47.42 \pm 7.482^{*}$ & $43.85 \pm 0.835$ \\
\hline Asp & $23.47 \pm 1.864$ & $12.20 \pm 0.852^{*}$ & $20.32 \pm 0.831$ \\
\hline Glu & $56.26 \pm 3.580$ & $45.33 \pm 2.548$ & $53.32 \pm 2.398$ \\
\hline Thr & $56.99 \pm 4.217$ & $28.74 \pm 1.893^{*}$ & $38.07 \pm 0.367$ \\
\hline Pro $+\beta$-Ala & $191.5 \pm 48.20$ & $95.33 \pm 12.73$ & $108.9 \pm 4.532$ \\
\hline Gly & nd & nd & $2.121 \pm 2.121$ \\
\hline GABA & $9.563 \pm 0.906$ & nd & nd \\
\hline Ala & $44.05 \pm 14.03$ & $30.01 \pm 13.08^{*}$ & $27.24 \pm 1.487$ \\
\hline Val & $31.73 \pm 1.724$ & $16.41 \pm 1.419^{*}$ & $27.78 \pm 0.292$ \\
\hline Met & nd & nd & nd \\
\hline Leu & $5.003 \pm 0.835$ & $3.632 \pm 0.772$ & $4.583 \pm 0.140$ \\
\hline Ile & nd & $7.053 \pm 1.629^{*}$ & $14.00 \pm 0.478$ \\
\hline Trp & $10.92 \pm 1.724$ & $4.082 \pm 0.623^{*}$ & $9.982 \pm 0.032$ \\
\hline Orn & nd & nd & nd \\
\hline Phe & $15.74 \pm 2.173$ & $7.379 \pm 0.592^{*}$ & $16.10 \pm 0.067$ \\
\hline Lys & $5.084 \pm 0.492$ & $3.466 \pm 0.520$ & $6.030 \pm 0.328$ \\
\hline Cys & $4.573 \pm 0.324$ & nd & nd \\
\hline Tyr & $12.31 \pm 0.898$ & $9.252 \pm 0.433^{*}$ & $11.14 \pm 0.032$ \\
\hline TFAA & $1,357.9 \pm 278.6$ & $683.6 \pm 129.4^{*}$ & $691.4 \pm 7.443$ \\
\hline
\end{tabular}

${ }^{*} p<0.05$ (Between raw state and boiled state).

in spinach after boiling and the lost amino acids seemed to appear in the cooking liquid (Table 7). However, GABA and Met disappeared after the boiling treatment and were absent even in the cooking liquid. It would be ideal for the purpose of nutritional values if boiled spinach were eaten with cooking liquid. However, this is not realistic since cooking liquid contains oxalic acid, which results in poor palatability. Hence it is recommended to eat boiled spinach together with other foods that contain much amino acids.

\section{Komatsuna}

Major free amino acids in komatsuna were Gln and Pro $(+\beta$-Ala). Other free amino acids were also present. After boiling, komatsuna lost about half of its free amino acids. GABA and Cys were absent, as their levels were below the detection limit, whereas 12 amino acids such as His, Arg, Asn, and Trp showed less than 50\% retention after boiling $(p<0.05)$ (Table 8). A significant amount of free amino acids was detected in the cooking liquid, probably owing to the extraction of these 12 amino acids after boiling treatment, resulting in the lowering of the nutritional value of komatsuna.

Vegetables are good sources of free amino acids. After consumption of vegetables, free amino acids are released during digestion and absorbed by the digestive system. Practically, not all vegetables are eaten raw, and most of these are cooked by boiling and/or baking. Hence, it is important to determine the amount and type of free amino acids sensitive to the cooking methods.

About $30-80 \%$ of free amino acids in vegetables were intact after boiling. Snap peas maintained nearly $80 \%$ of free amino acids present in uncooked samples, whereas Western carrot and cabbage lost most of these free amino acids.

It is evident that the behavior of these amino acids differed with respect to the cooking method employed. The most-affected amino acids were Asn, Gln, and GABA, which tended to disappear, probably through metabolism or destruction.

The contents of certain amino acids such as GABA and Glu were changed upon heating of some vegetables such as eggplants or tomatoes (6). Glutamate decarboxylase $(\mathrm{GAD})$ is the enzyme responsible for raising GABA contents after steaming vegetables (11). A previous study employed three cooking methods for bamboo shoot cooking and compared the content of amino acids (7). About 40 to $80 \%$ of amino acids were retained in their free forms after boiling, stir-frying, or steaming. Boiling resulted in a substantial decrease in total amino acid contents (7). In our study, similar results were obtained, but boiling resulted in a decrease in the free amino acid contents. We observed a similar tendency and the lost free amino acids after boiling were recovered from the cooking liquids (Tables 5 to 8 ).

The results of the present study highlight the changes in the contents of free amino acids during cooking with 
methods that are typically used on a daily basis. Furthermore, our study on the dynamics of free amino acids caused by various cooking methods should provide ample information for future nutritional studies.

\section{Acknowledgments}

We would like to thank Editage (www.editage.jp) for English language editing.

\section{REFERENCES}

1) Fabbri AD, Crosby GA. 2016. A review of the impact of preparation and cooking on the nutritional quality of vegetables and legumes. Int J Gastron Food Sci 3: 2-11.

2) Miglio C, Chiavaro E, Visconti A, Fogliano V, Pellegrini N. 2008. Effects of different cooking methods on nutritional and physicochemical characteristics of selected vegetables. J Agr Food Chem 56: 139-147.

3) Yuan GF, Sun B, Yuan J, Wang QM. 2009. Effects of different cooking methods on health-promoting compounds of broccoli. J Zhejiang Univ Sci B 10: 580-588.

4) Turkmen N, Sari F, Velioglu YS. 2005. The effect of cooking methods on total phenolics and antioxidant activity of selected green vegetables. Food Chem 93: 713-718.

5) Kasai M, Ishiguro K, Kyouda H, Hamazono T, Hatae K,
Shimada A. 2000. Change in the amounts of reducing sugars and free amino acids in rice during the cooking processes. J Home Econ Jpn 51: 579-585 (in Japanese).

6) Horie H. 2012. Production of guanylic acid by heating vegetables. J Cookery Sci Jpn 45: 346-351 (in Japanese).

7) Zhang JJ, Ji R, Hu YQ, Chen JC, Ye XQ. 2011. Effect of three cooking methods on nutrient components and antioxidant capacities of bamboo shoot (Phyllostachys praecox C.D. Chu et C.S. Chao). J Zhejiang Univ Sci B 12: 752-759.

8) Ito H, Ueno H. 2014. Construction of free amino acids composition data base for food. J Chem Chem Eng 8: 501-515.

9) Ito H, Ueno H, Kikuzaki H. 2017. Construction of a free-form amino acid database for vegetables and mushrooms. Integr Food Nutr Metab 4: 1-9.

10) Verkerk R, Dekker M. 2004. Glucosinolates and myrosinase activity in red cabbage (Brassica oleracea L. var. Capitata f. rubra DC.) after various microwave treatments. J Agric Food Chem 52: 7318-7323.

11) Yaghi S, Otoguro C, Sumino T, Hara H, Kaneko K. 2008. Effect of low-temperature stream heating on the free amino acids in various vegetables. J Cookery Sci Jpn 41: 42-48 (in Japanese). 